Supporting Information for

\title{
Mesoporous PdRu Nanocrystals for Oxygen Reduction Electrocatalysis
}

Hongjing Wang, Tongqing Zhou, Kai Deng, Shiqian Jiao, Wenjing Tian, You Xu, Xiaonian Li,

Ziqiang Wang*, and Liang Wang*

State Key Laboratory Breeding Base of Green-Chemical Synthesis Technology, College of Chemical Engineering, Zhejiang University of Technology, Hangzhou 310014, P. R. China.

\section{Corresponding authors}

*E-mail: zqwang@zjut.edu.cn (Z.W.)

*E-mail: wangliang@zjut.edu.cn (L.W.) 

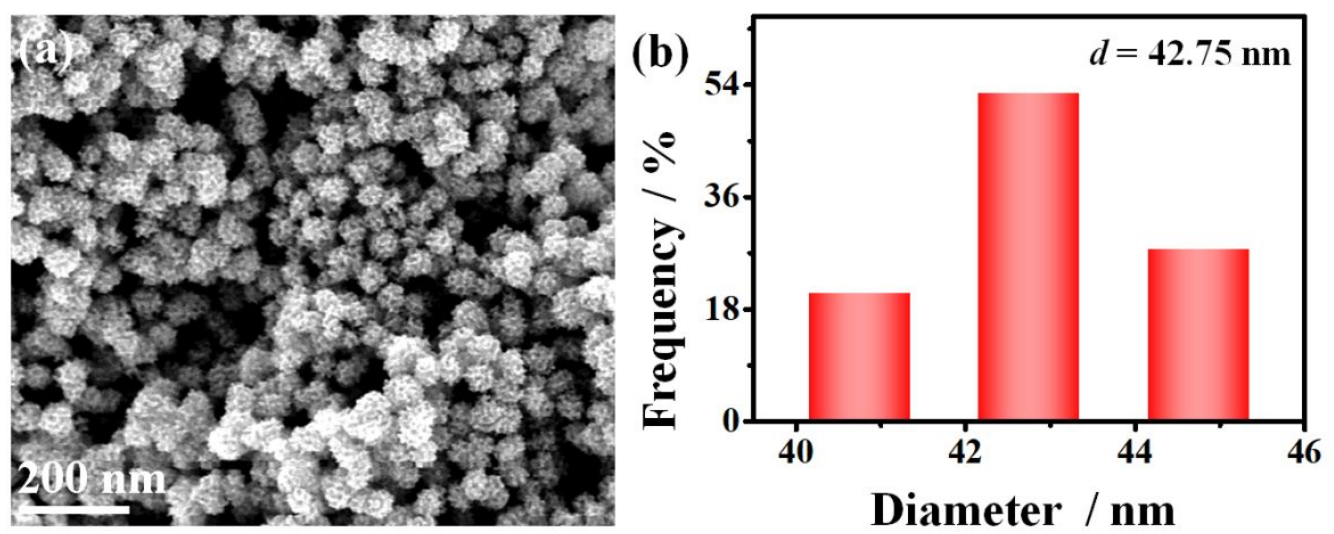

Figure S1. (a) SEM image and (b) particle size distribution of the PdRu MNCs.

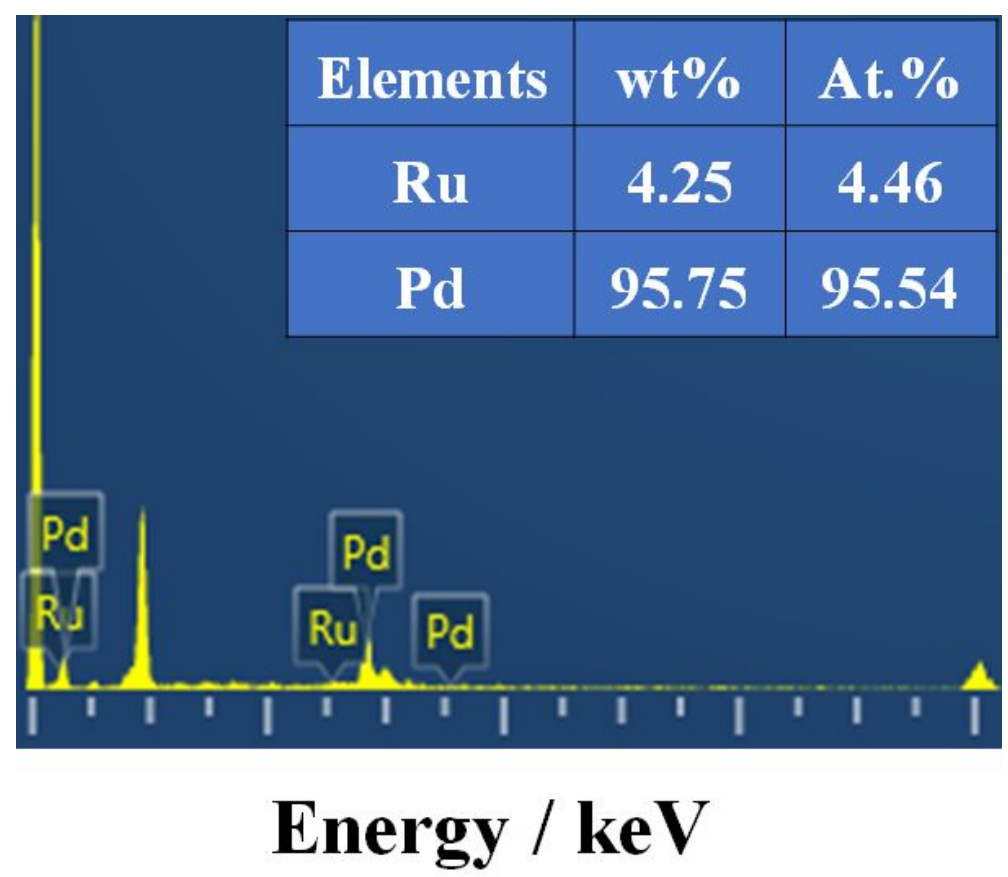

Figure S2. EDX spectrum of the PdRu MNCs. 


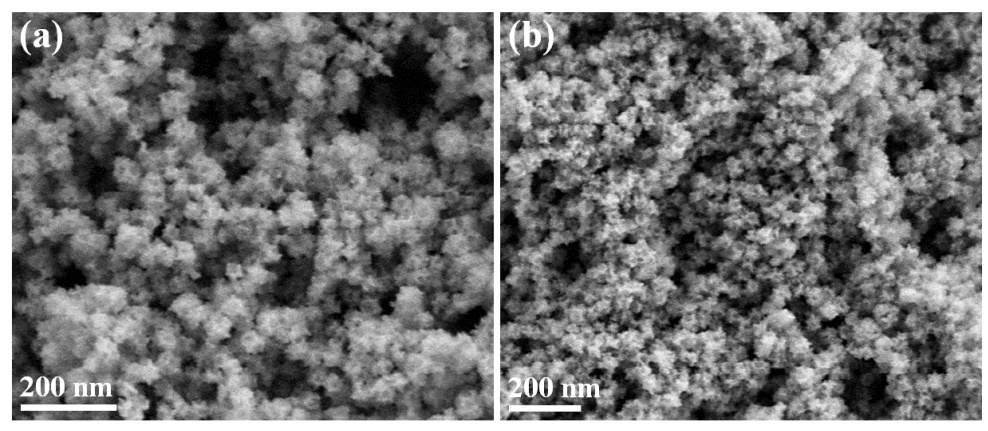

Figure S3. SEM images of PdRu MNCs prepared at (a) $60{ }^{\circ} \mathrm{C}$ and (b) $80^{\circ} \mathrm{C}$.
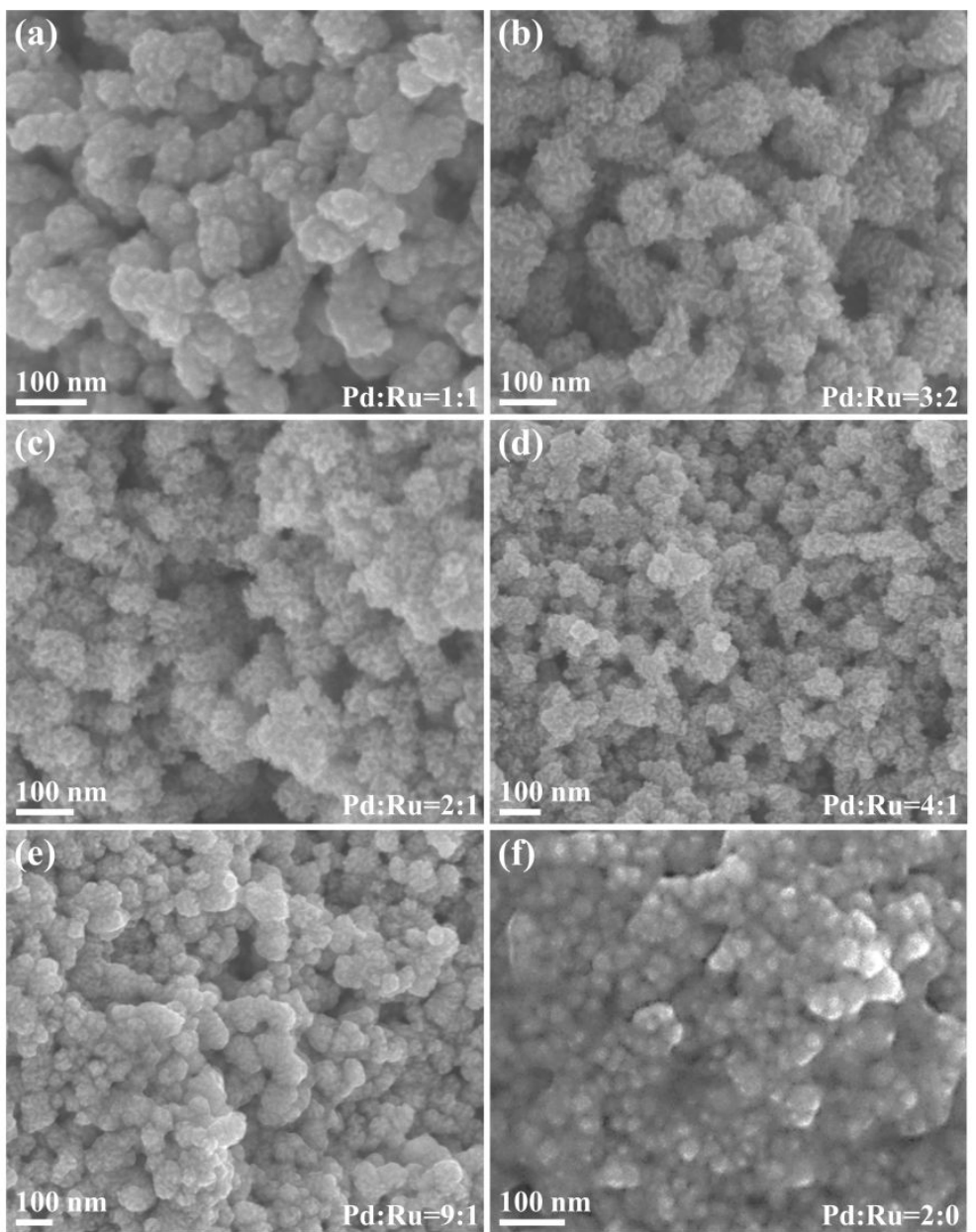

Figure S4. SEM images of the products prepared with different contents of precursors: (a) $1 \mathrm{~mL}$ $\mathrm{Na}_{2} \mathrm{PdCl}_{4}+1 \mathrm{~mL} \mathrm{RuCl}_{3}$ (b) $1.2 \mathrm{~mL} \mathrm{Na}_{2} \mathrm{PdCl}_{4}+0.8 \mathrm{~mL} \mathrm{RuCl}$, (c) $1.33 \mathrm{~mL} \mathrm{Na}_{2} \mathrm{PdCl}_{4}+0.67 \mathrm{~mL}$ $\mathrm{RuCl}_{3}$, (d) $1.6 \mathrm{~mL} \mathrm{Na}_{2} \mathrm{PdCl}_{4}+0.4 \mathrm{~mL} \mathrm{RuCl}_{3}$, and (e) $1.8 \mathrm{~mL} \mathrm{Na}_{2} \mathrm{PdCl}_{4}+0.2 \mathrm{~mL} \mathrm{RuCl}_{3}$, and (f) 2.0 
$\mathrm{mL} \mathrm{Na}{ }_{2} \mathrm{PdCl}_{4}$.
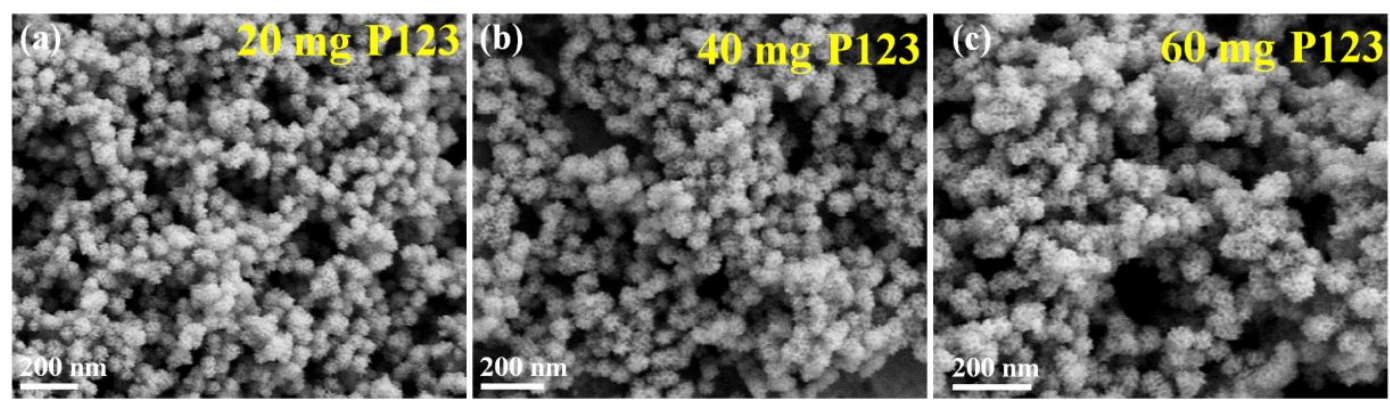

Figure S5. SEM images of the PdRu samples prepared under the typical synthetic conditions with different surfactants: (a) $20 \mathrm{mg}$ P123, (b) $40 \mathrm{mg}$ P123, and (c) $60 \mathrm{mg}$ P123.

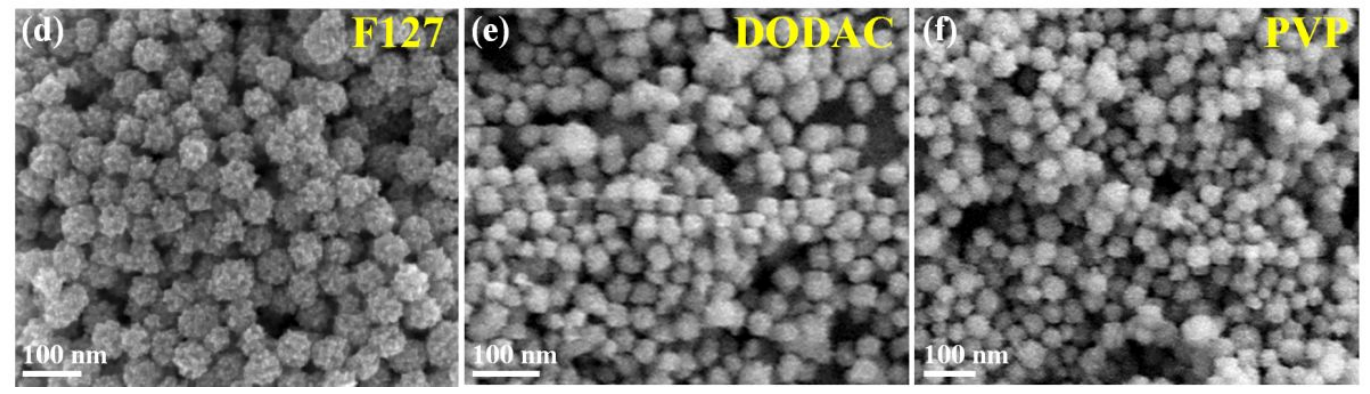

Figure S6. SEM images of the PdRu samples prepared under the typical synthetic conditions with different surfactants: (a) F127, (b) DODAC, and (c) PVP. 

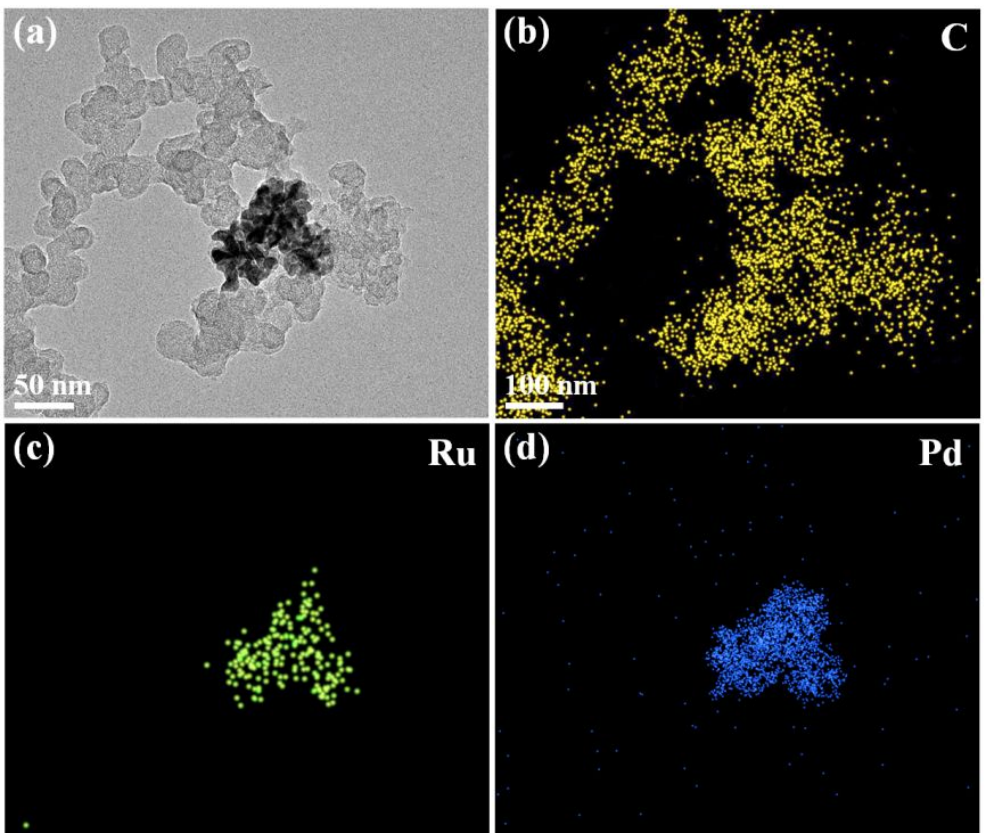

Figure S7. (a) TEM image and (b-d) corresponding elemental mapping images of PdRu MNCs/C.
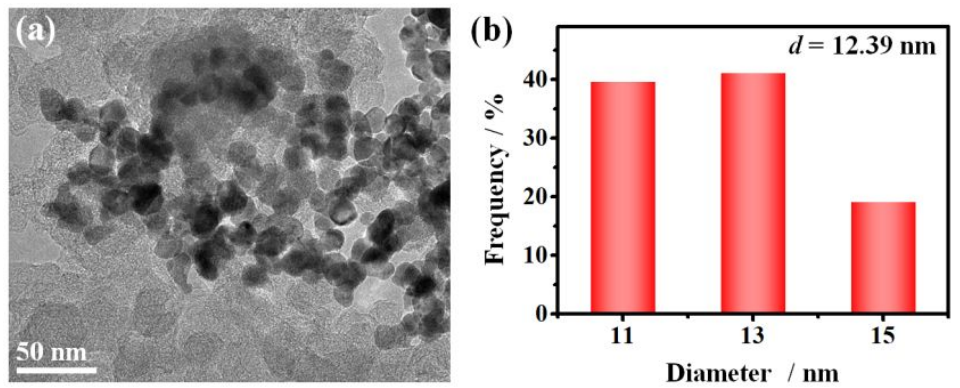

Figure S8. (a) TEM image of Pd NPs/C and (b) particle size distribution of Pd NPs. 

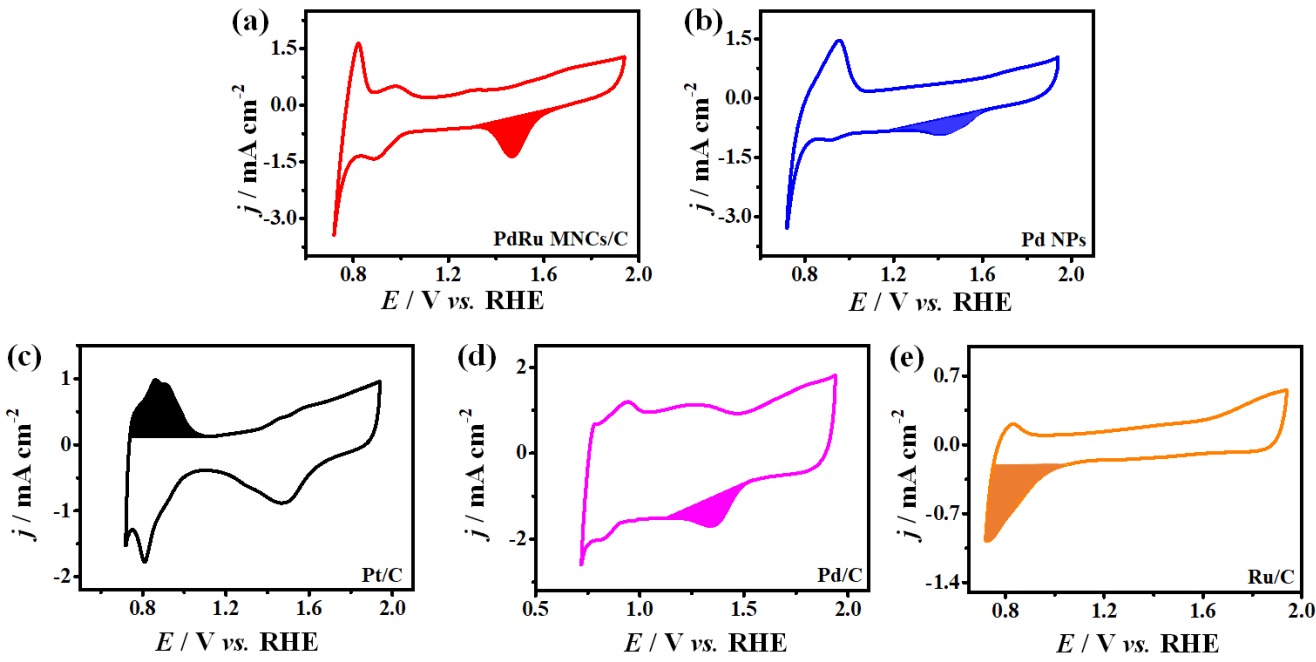

Figure S9. CV curves for different catalysts in $0.1 \mathrm{M} \mathrm{HClO}_{4}$.
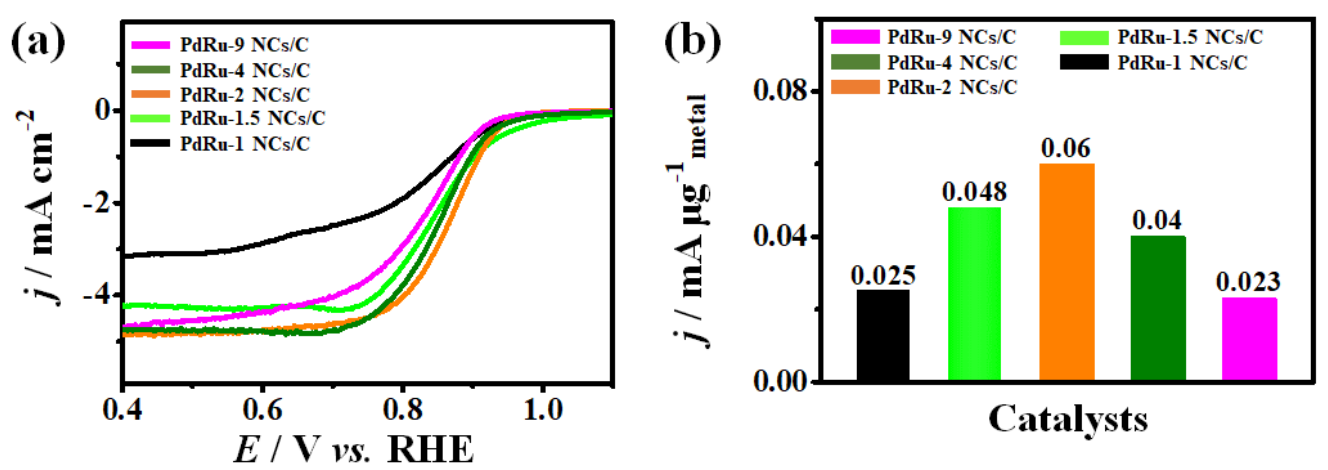

Figure S10. (a) ORR activity and (b) mass activity of samples prepared from different ratios of Pd and $\mathrm{Ru}$ precursors. 

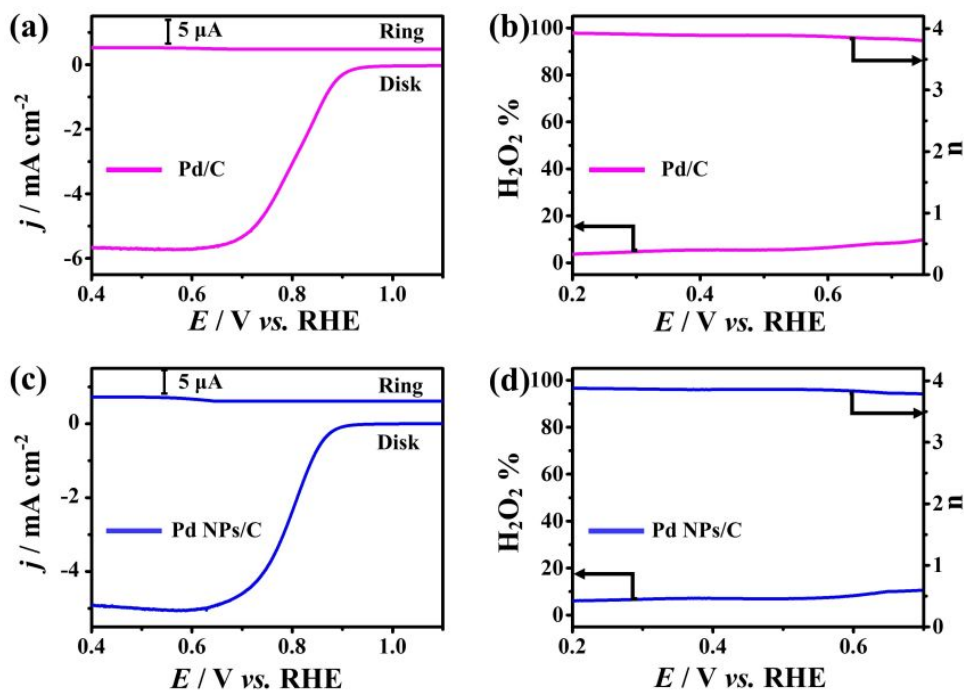

Figure S11. (a, c) RRDE test in an $\mathrm{O}_{2}$-saturated $0.1 \mathrm{M} \mathrm{KOH}$ solution with a rotation rate of 1600 rpm, and $(b, d)$ measured peroxide percentage and electron transfer number.
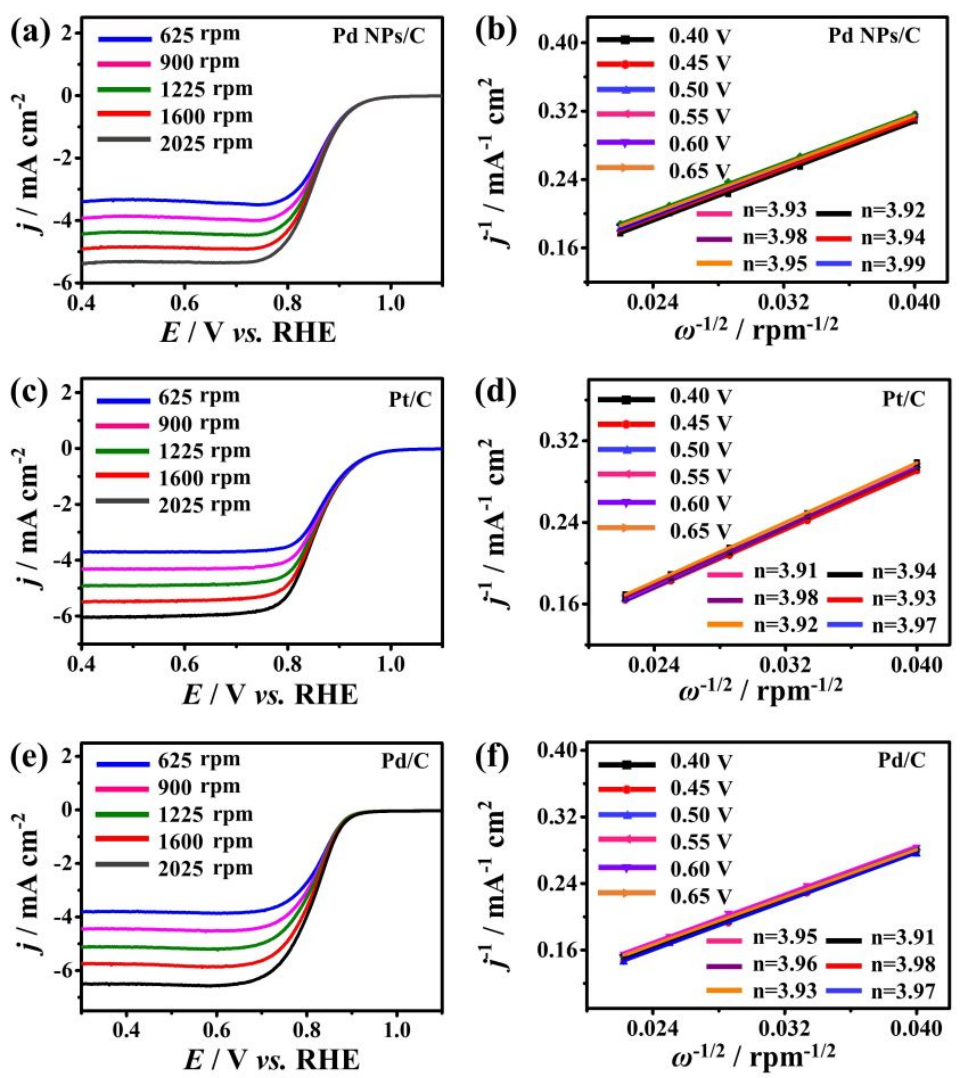

Figure S12. (a, c and e) Rotation-rate-dependent current-potential curves of catalysts, and (b, d, and f) corresponding electron transfer number at different potentials. 

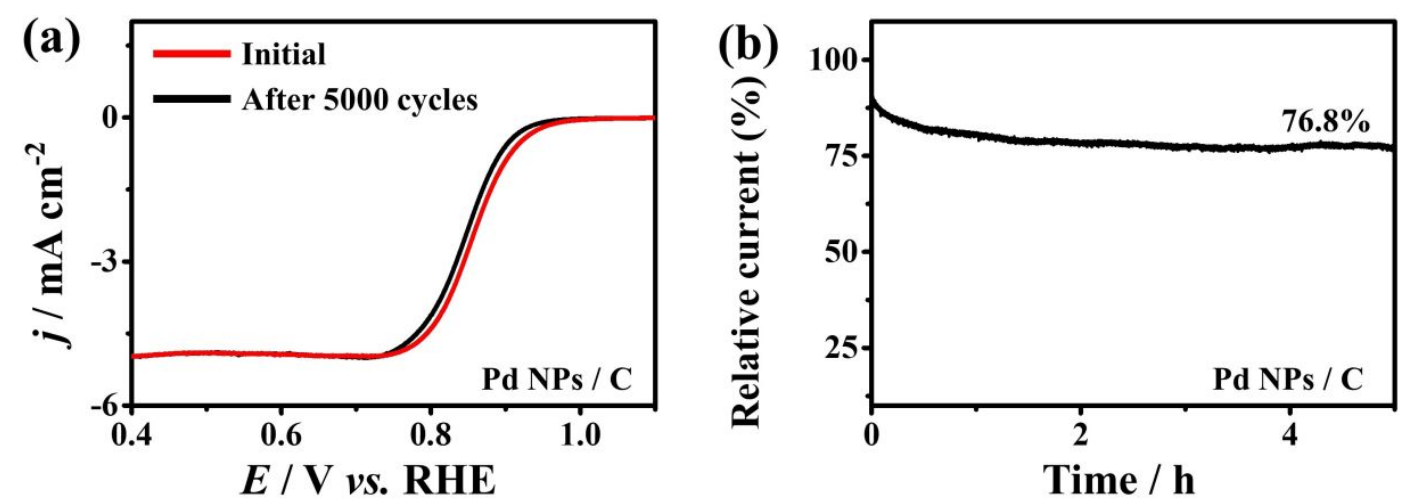

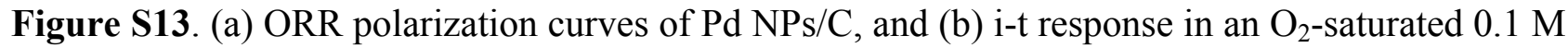
$\mathrm{KOH}$ solution with a rotation rate of $1600 \mathrm{rpm}$.
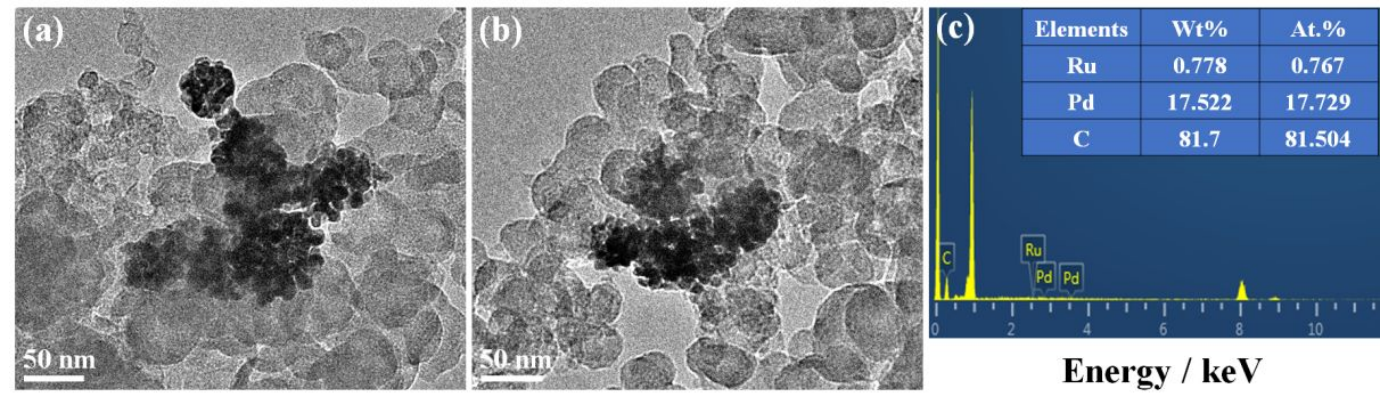

Figure S14. (a) TEM image of the PdRu MNCs/C and (b) TEM image and (c) corresponding EDX spectrum of the PdRu MNCs/C after durability test. 
Table S1. The ORR performance comparison of PdRu MNCs with published works in the same condition.

\begin{tabular}{|c|c|c|c|}
\hline Catalysts & $\begin{array}{l}\text { Mass activities } \\
\qquad\left(\mathrm{mA} \mu \mathrm{g}_{\mathbf{P d}}{ }^{-1}\right)\end{array}$ & $\begin{array}{l}\text { Specific activities } \\
\qquad\left(\mathrm{mA} \mathrm{cm}^{-2}\right)\end{array}$ & References \\
\hline PdRu MNCs/C & 1.55 & 2.90 & This work \\
\hline $\mathrm{Pd}_{3} \mathrm{~Pb} / \mathrm{Pd} \mathrm{NSs} / \mathrm{C}$ & 0.57 & 1.31 & 1 \\
\hline Ordered $\mathrm{Pd}_{3} \mathrm{Fe} / \mathrm{C}$ & 0.09 & l & 2 \\
\hline $\mathrm{PdCuCo} \mathrm{NPs} / \mathrm{C}$ & 0.13 & l & 3 \\
\hline $\mathrm{Pd}_{3} \mathrm{~Pb}$ tripods $/ \mathrm{C}$ & 0.56 & 1.76 & 4 \\
\hline $\mathrm{Pd} / \mathrm{W}_{18} \mathrm{O}_{49}$ hybrids & 0.22 & 0.22 & 5 \\
\hline $\mathrm{Pd}_{6} \mathrm{Ni}$ icosahedra & 0.22 & l & 6 \\
\hline $\mathrm{Pd}_{3} \mathrm{Bi} / \mathrm{C}$ NPs & 1.20 & 2.30 & 7 \\
\hline $\mathrm{Pd}_{31} \mathrm{Bi}_{12} / \mathrm{C}$ NPs & 0.95 & 2.45 & 8 \\
\hline $\mathrm{Pd} / \mathrm{PdFe} \mathrm{NPs}$ & 0.31 & 1.56 & 9 \\
\hline Au-NWs@Pd@PEI & 0.295 & / & 10 \\
\hline
\end{tabular}




\section{References}

(1) Tang, C.; Zhang, N.; Ji, Y.; Shao, Q.; Li, Y.; Xiao, X.; Huang, X. Fully Tensile Strained $\mathrm{Pd}_{3} \mathrm{~Pb} / \mathrm{Pd}$ Tetragonal Nanosheets Enhance Oxygen Reduction Catalysis. Nano Lett. 2019, 19 (2), 1336-1342, DOI: 10.1021/acs.nanolett.8b04921.

(2) Cui, Z.; Li, L.; Manthiram, A.; Goodenough, J. B. Enhanced Cycling Stability of Hybrid Li-Air Batteries Enabled by Ordered $\mathrm{Pd}_{3} \mathrm{Fe}$ Intermetallic Electrocatalyst. J. Am. Chem. Soc. 2015, 137 (23), 7278-81, DOI: 10.1021/jacs.5b03865.

(3) Jiang, K.; Wang, P.; Guo, S.; Zhang, X.; Shen, X.; Lu, G.; Su, D.; Huang, X. Ordered $\mathrm{PdCu}$-Based Nanoparticles as Bifunctional Oxygen-Reduction and Ethanol-Oxidation Electrocatalysts. Angew. Chem., Int. Ed. 2016, 55 (31), 9030-9035, DOI: 10.1002/anie.201603022.

(4) Bu, L.; Shao, Q.; Pi, Y.; Yao, J.; Luo, M.; Lang, J.; Hwang, S.; Xin, H.; Huang, B.; Guo, J.; Su, D.; Guo, S.; Huang, X. Coupled s-p-d Exchange in Facet-Controlled $\mathrm{Pd}_{3} \mathrm{~Pb}$ Tripods Enhances Oxygen Reduction Catalysis. Chem 2018, 4 (2), 359-371, DOI: 10.1016/j.chempr.2018.01.002.

(5) Lu, Y.; Jiang, Y.; Gao, X.; Wang, X.; Chen, W. Strongly Coupled Pd Nanotetrahedron/Tungsten Oxide Nanosheet Hybrids with Enhanced Catalytic Activity and Stability as Oxygen Reduction Electrocatalysts. J. Am. Chem. Soc. 2014, 136 (33), 11687-97, DOI: 10.1021/ja5041094.

(6) Feng, Y. G.; Shao, Q.; Ji, Y. J.; Cui, X. N.; Li, Y. Y.; Zhu, X.; Huang, X. Q. Surface-Modulated Palladium-nickel Icosahedra as High-Performance Non-Platinum Oxygen Reduction Electrocatalysts. Sci. $A d v$. 2018, 4 (7), eaap8817, DOI: 10.1126/sciadv.aap8817.

(7) Sun, D.; Wang, Y.; Livi, K. J. T.; Wang, C.; Luo, R.; Zhang, Z.; Alghamdi, H.; Li, C.; An, F.; S10 
Gaskey, B.; Mueller, T.; Hall, A. S. Ordered Intermetallic $\mathrm{Pd}_{3} \mathrm{Bi}$ Prepared by an Electrochemically Induced Phase Transformation for Oxygen Reduction Electrocatalysis. ACS Nano 2019, 13 (9), 10818-10825, DOI: 10.1021/acsnano.9b06019.

(8) Wang, Y.; Hall, A. S. Pulsed Electrodeposition of Metastable $\operatorname{Pd}_{31} \mathrm{Bi}_{12}$ Nanoparticles for Oxygen Reduction Electrocatalysis. ACS Energy Lett. 2019, 5 (1), 17-22, DOI: 10.1021/acsenergylett.9b02219.

(9) Li, X.; Li, X.; Liu, C.; Huang, H.; Gao, P.; Ahmad, F.; Luo, L.; Ye, Y.; Geng, Z.; Wang, G.; Si, R.; Ma, C.; Yang, J.; Zeng, J. Atomic-Level Construction of Tensile-Strained PdFe Alloy Surface toward Highly Efficient Oxygen Reduction Electrocatalysis. Nano Lett. 2020, 20 (2), 1403-1409, DOI: 10.1021/acs.nanolett.9b05024.

(10) Xue, Q.; Bai, J.; Han, C.; Chen, P.; Jiang, J.-X.; Chen, Y. Au Nanowires@Pd-Polyethylenimine Nanohybrids as Highly Active and Methanol-Tolerant Electrocatalysts toward Oxygen Reduction Reaction in Alkaline Media. ACS Catal. 2018, 8 (12), 11287-11295, DOI: 10.1021/acscatal.8b03447. 